\title{
Nutritional Risk Assessment at a Municipal Hospital Using Nutritional Tools: A Retrospective Study
}

\author{
Junichi Araki ${ }^{1}$, Yutaka Inoue ${ }^{2, *}$, Lina Honda ${ }^{2}$, Shiho Morita², Munechika Tsuchiya ${ }^{3}$, Taku Ueno ${ }^{1}$, and Ikuo Kanamoto ${ }^{2}$ \\ ${ }^{1}$ Department of Pharmacy, IMS Fujimi General Hospital, Japan \\ ${ }^{2}$ Laboratory of Drug Safety Management, Faculty of Pharmacy and Pharmaceutical Sciences, Japan \\ ${ }^{3}$ Department of Nutrition, IMS Fujimi General Hospital, Japan
}

*Corresponding author: Yutaka Inoue, Laboratory of Drug Safety Management, Faculty of Pharmacy and Pharmaceutical Sciences, Josai University, 1-1 Keyakidai, Sakado-shi, Saitama, 3500295, Japan, Tel: +81-49-271-7317; Fax: +81-49-271-7317; E-mail:

yinoue@josai.ac.jp

Received: 05 Dec, 2019 | Accepted: 24 Dec, 2019 | Published: 06 Jan, 2020

Citation: Araki J, Inoue Y, Honda L, Morita S, Tsuchiya M, et al. (2020) Nutritional Risk Assessment at a Municipal Hospital Using Nutritional Tools: A Retrospective Study. J Drug Res Dev 6(1): dx.doi.org/10.16966/2470-1009.149

Copyright: (c) 2019 Araki J, et al. This is an open-access article distributed under the terms of the Creative Commons Attribution License, which permits unrestricted use, distribution, and reproduction in any medium, provided the original author and source are credited.

\begin{abstract}
Background and Aims: This study aimed to determine how nutritional endpoints affected nutritional risk assessment. We conducted nutritional screening using 3 different tools, namely, the modified SGA (mSGA), MUST, and Mini Nutritional Assessment ${ }^{\circledR}$ Short-Form (MNA ${ }^{\circledR}$-SF). We compared the results of these nutritional evaluation tools and compared the parameters evaluated.

Methods: This retrospective study comprised 1253 patients (mean age $71.0 \pm 15.1$ years) who had been admitted to the IMS Fujimi General Hospital from October to December 2017 (excluding pediatric admissions). Based on patient electronic medical records, nutritional status was determined using the abovementioned assessment tools. Additionally, we considered the parameters used in each evaluation tool and examined their use in determining nutritional status.

Results: The mSGA, MUST, and MNA ${ }^{\circledR}-$ SF tools indicated that $15.1 \%, 31.4 \%$, and $24.2 \%$ of patients were malnourished, respectively. Among the parameters affecting screening outcomes, assessing BMI was used in all tools. Moreover, pressure injury and mobility were found to be frequently used as evaluation parameters in the $\mathrm{mSGA}$ and the $\mathrm{MNA}^{\circledR}-\mathrm{SF}$, respectively. Compared with the mSGA, the MUST and the MNA ${ }^{\circledR}$-SF evaluated the presence of malnutrition more frequently.

Conclusions: BMI assessment affects the nutritional status of a patient and was an essential factor in nutritional assessment, based on the 3 tools employed in our study. The $\mathrm{MNA}^{\circledR}$-SF appeared more readily usable for nutritional risk assessment because of smaller evaluation parameter numbers and being easier to apply than the MSGA. The MNA ${ }^{\circledR}-S F$ also included walking unaided as an evaluation parameter for nutritional status alongside diet and weight loss.
\end{abstract}

Keywords: Nutritional risk; Nutritional screening; SGA; MUST; MNA ${ }^{\circledR}-S F ;$ BMI

\section{Introduction}

The concept of creating a Nutritional Support Team (NST) originated from the United States in the 1970s and has been gradually adopted in Japan from 1998. An NST is comprised of doctors, nurses, pharmacists, registered dietitians, laboratory technicians, physiotherapists, and other relevant healthcare personnel. Nutritional management with an NST is ideal for those patients requiring such management to ensure optimal health outcomes. However, intervention and nutrition management for all patients requires a great deal of time and is costly; therefore, it is impractical to use for all patients. In clinical practice, nutritional screening is used to identify patients with malnutrition, particularly those at risk of malnutrition, who will require NST intervention. In nutritional assessment screening, a nutritional assessment tool is useful to predict the nutritional status of a patient. The nutritional status is known to have various effects on patient prognosis. Malnutrition reduces immunity and interferes with healing [1]. Moreover, the incidence of complications increases with malnutrition, the length of hospital stay is extended [2], and hospitalization costs increase [3]. Managing the nutritional status can prevent an increase in the incidence of these complications. Several studies have reported that NST interventions have resulted in positive outcomes, such as a reduction in the length of hospitalization, and a decline in the mortality rate [4,5]. Hence, the implementation of nutritional management through the collaborative efforts of healthcare teams is likely to be an important factor in providing effective healthcare. 
Currently, several nutritional assessment tools have been proposed, using a variety of techniques. The Subjective Global Assessment (SGA) tool, devised by Destky AS, et al. in 1987, consists of a simple interview and physical findings [6]. Nutritional management according to the SGA has been reported to correlate positively with postoperative complication rates and the average length of hospitalization of hospitalized patients in the acute phase [7-9]. The Malnutrition Universal Screening Tool (MUST) is a tool proposed by the British Association for Parenteral and Enteral Nutrition (BAPEN). The MUST uses a stepwise procedure in its assessment, with BMI assessed in a first step, and weight loss and food intake over the following 5 days assessed in second and third steps, respectively. The parameters for each step are evaluated using 3 score levels $(0,1$, and 2) [10]. Nutritional interventions using the MUST have been reported to correlate positively with the length of hospital stay among patients who were elderly and among those with cancer $[11,12]$.

The Mini Nutritional Assessment ${ }^{\oplus}\left(\mathrm{MNA}^{\oplus}\right)$, developed for elderly people ( $\geq 65$ years old), uses a total of 18 evaluation items, including dysphagia and related impaired cognitive function that are often found in elderly people, and takes into account the degree of independence in daily life. Nutritional status is determined in terms of whether nutrition is good, at risk, or poor [13]. In addition, the Mini Nutritional Assessment ${ }^{\circ}$-Short Form (MNA ${ }^{\circ}$-SF) has been developed, which comprises 6 evaluation items considered as the most important parameters in the $\mathrm{MNA}^{\circ}$ [14]. The MNA ${ }^{\circ}-\mathrm{SF}$ has been found to correlate positively with the $\mathrm{MNA}^{\bullet}[15]$, and it has been reported that even elderly Japanese patients can adequately identify malnourished patients [16,17]. Other nutritional evaluation tools have been proposed, and appropriate tools are selected depending on the patient background within specific medical facilities.

In recent years, the Japanese Society for Parenteral and Enteral Nutrition (JSPEN) has recommended that SGA nutritional screening be performed on all patients. The SGA is based on the subjective assessment of the evaluator. Therefore, to use the SGA to obtain accurate results, appropriate education of the nutrition evaluator is needed. Therefore, at the IMS Fujimi General Hospital (Saitama, Japan), a modified version of the SGA, the mSGA, has been created and used for nutritional screening (Table 1). The mSGA is used to screen for malnutrition patients conducted in all patients.

The mSGA has some limitations, primarily due to patient low awareness levels, which means that care workers cannot readily acquire sufficiently accurate patient information. When also considering the numerous evaluation items of the mSGA and the complexity of undertaking nutritional management in relation to it, these factors have been deemed to lead to misunderstandings of a patient's nutritional status by an evaluator, with direct effects on the quality of the nutritional evaluation. It is possible that underestimation may occur concerning the nutritional risks for patients in need of nutritional management. Given this situation, it would appear advantageous if the nutritional evaluation items of the mSGA could be reduced to create a set of more straightforward and useful items, so that the evaluation of patients' nutritional status in the IMS Fujimi General Hospital could be more effectively executed. In addition, although mSGA was established in the hospital after discussions with each medical staff, it has not been compared with other nutritional assessment tools, and it is considered necessary to improve it.

Therefore, we aimed to determine how nutritional endpoints affect nutritional risk assessment. The purpose of this study was to consider whether the comparison of nutrition evaluation tools can be used at IMS Fujimi General Hospital and other medical institutions. Based on the results of mSGA evaluation and medical record information, we conducted nutritional screening using the MUST and the MNA ${ }^{\circ}$ $\mathrm{SF}$, and we verified our results in relation to these nutrition evaluation tools and their corresponding evaluation items through comparison and examination (Table 1).

\section{Materials and Methods}

\section{Participants}

A total of 1253 patients ( 728 males, 525 females, excluding pediatric patients) who had been admitted to the IMS Fujimi General Hospital over 3 months (October to December 2017), with an average age of $71.0 \pm 15.1$ years, were considered participants of this study.

\section{Initial screening of nutritional status on admission using the mSGA}

The mSGA, a tool based on the SGA and modified at the IMS Fujimi General Hospital, was used to assess patient nutritional risk. The following patient profile data (age, height, weight, BMI, and serum albumin level) recorded on admission were obtained from the hospital's electronic medical record database and were used in the assessment. As an exception, when there was no record of height data and BMI could not be calculated, or when serum albumin levels had not been measured, the IMS Fujimi General Hospital stipulated that points should be added to avoid a risk of underestimation. This study conducted an evaluation based on this data. Furthermore, further factors including dietary conditions, aggregated weight change, vomiting, diarrhea, fever, dehydration, swallowing state, tube feeding and enteral nutrition, and decubitus ulceration were used as parameters in the nutritional assessment. Based on the tabulated results, nutritional risk assessment was performed using the mSGA nutritional assessment items, with pediatric patients excluded as the mSGA is a tool intended for adults only.

\section{Nutritional risk assessment using the MUST}

Since the mSGA constitutes a large number of evaluation items (9 items) and is complex to use, nutritional risk was evaluated using the MUST (3 items) (Table 2). Given that each step was evaluated with

Table 1: The modified Subjective Global Assessment (mSGA) nutrition screening tool used in the IMS Fujimi General Hospital.

\begin{tabular}{|l|l|c|}
\hline \multicolumn{1}{|c|}{ Content } & Score \\
\hline A. & Decrease in food intake during the last 5 days & 1 \\
\hline B. & Weight loss of $3 \mathrm{~kg}$ or more during the last 6 months & 1 \\
\hline C. & Repeat diarrhea/ vomiting the last 5 days & 1 \\
\hline D. & Fever of $37.0^{\circ} \mathrm{C}$ or more or dehydration symptoms & 1 \\
\hline E. & History of aspiration pneumonia or choking on water & 1 \\
\hline F. & Tube feeding & 1 \\
\hline G. & Pressure ulcers & 3 \\
\hline H. & BMI less than $18.5 \mathrm{~kg} / \mathrm{m}^{2}$ & 1 \\
\hline I. & $\begin{array}{l}\text { Serum albumin value within the past month is } 3.0 \\
\text { g/dL or less }\end{array}$ & 1 \\
\hline Add score A-I to calculate risk of malnutrition \\
\hline Total Score & Risk of malnutrition \\
\hline 0 & No risk \\
\hline $1-2$ & Low risk \\
\hline $3-5$ & Medium risk \\
\hline 6 or more & High Risk \\
\hline
\end{tabular}


reference to the mSGA, each step was assigned the relevant mSGA evaluation item as follows. This study is a retrospective study, in which the mSGA items are interpreted and assigned to the MUST items as follows. Regarding 'Step 1: BMI score', the BMI was calculated using height and weight taken from patient basic data and then evaluated. In Step 2, the item 'Unintended weight loss in the past 3 to 6 months' was evaluated as having 1 point when it corresponded to the mSGA evaluation item 'Item 2: weight loss' and having 0 points when not applicable. For Step 3, the mSGA endpoint 'presence of an acute disease that might impair nutrition or 5 days' was evaluated as having 2 points when corresponding to 'Item 1 diet decrease.'

In MUST guidelines issued by BAPEN, the third step focuses on a patient's meal situation in relation to dysphagia due to head trauma that may affect cerebral function [10]. However, dysphagia in the mSGA is not an acute symptom. Therefore, this study used the 'meal reduction' item in the $\mathrm{mSGA}$, which corresponds with 'no more or less than half a meal for 5 days.' Based on the evaluation results of each step, the nutritional status at each level was assessed and the evaluation items were summarized. In addition, we compared these findings with nutritional risk assessments derived from the mSGA (Table 2).

\section{Nutritional risk assessment using the $\mathrm{MNA}^{\oplus}$-SF}

The evaluation of patient data at admission was verified using the $\mathrm{MNA}^{\circ}$-SF, a nutritional risk assessment tool created for elderly people with fewer items than the mSGA (Table 3). In addition, this study is a retrospective study in which items in mSGA are interpreted and assigned to each item in MUST as follows.

The evaluation of 'Item A: The amount of food decreased due to anorexia, digestive problems, dysphagia, etc., in the past 3 months' corresponded to item 1 (meal reduction) and item 5 (growth disorder) in the mSGA. A score was set as 0 when either or both of items 1 and 5 corresponded, and as 2 when neither corresponded.

Table 2: The Malnutrition Universal Screening Tool (MUST).

\begin{tabular}{|l|l|c|}
\hline Step 1 & BMI score & \\
\hline & $>20$ (>30 Obese) & $=0$ \\
\hline & $18.5-20$ & $=1$ \\
\hline & $<18.5$ & $=2$ \\
\hline Step 2 & Weight loss score & \\
\hline & Unplanned weight loss in past 3-6 months & $=0$ \\
\hline & $<5 \%$ & $=1$ \\
\hline & $5-10 \%$ & $=2$ \\
\hline & $>10 \%$ & \\
\hline Step 3 & Acute disease effect score & $=2$ \\
\hline & $\begin{array}{l}\text { If patient is acutely ill and there has been or is } \\
\text { likely to be no nutritional intake for >5 days }\end{array}$ & \\
\hline Step 4 & $\begin{array}{l}\text { Add score of Step 1-3 to calculate overall risk of } \\
\text { malnutrition }\end{array}$ & Low risk \\
\hline & Score 0 & Medium risk \\
\hline & Score 1 & High risk \\
\hline & Score 2 or more & $\begin{array}{c}\text { Routine } \\
\text { clinical care }\end{array}$ \\
\hline Step 5 & Management guidelines & Observe \\
\hline & Low risk & Treat \\
\hline & Medium risk & \\
\hline & High risk & \\
\hline & &
\end{tabular}

'Item B: weight loss in the past 3 months' was determined using mSGA evaluation item 2 (weight loss). A score was set as 0 when item 2 corresponded with item $\mathrm{B}$, as 3 when there was no correspondence, and as 1 when the description was left blank. 'Item C: Can you walk by yourself?' was evaluated using information concerning the degree of daily life independence derived from patient basic data. 'Item D: mental stress and acute disease in the past 3 months' was determined using mSGA evaluation item 3 (diarrhea and vomiting) and item 4 (fever and dehydration). The score was set as 0 when either or both item 3 and item 4 corresponded to item D, and as 2 when neither item corresponded with item D. 'Item E: Neurological and mental problems' was determined from patient basic data in terms of whether restlessness, memory impairment, and the use of sleep agents and tranquilizers had been reported. When any one of these factors corresponded with item E, a score was set as 0 , and as 2 when there was no correspondence. Regarding 'Item F: BMI', this was calculated using height and weight information obtained from patient basic data.

Based on the evaluation results, nutritional status in terms of risk assessment using the evaluation items was tabulated. In addition, the results of the $\mathrm{MNA}^{\circ}$-SF were compared with those obtained using the mSGA (Table 3).

Table 3: The Mini Nutritional Assessment ${ }^{\circledR}$-Short Form (MNA ${ }^{\circledR}$-SF).

\begin{tabular}{|c|c|c|}
\hline \multirow[t]{2}{*}{ A } & \multicolumn{2}{|c|}{$\begin{array}{l}\text { Has food intake declined over the past } 3 \text { months due to } \\
\text { loss of appetite, digestive problems, chewing or swallowing } \\
\text { difficulties? }\end{array}$} \\
\hline & $0=$ & severe decrease in food intake \\
\hline & $1=$ & moderate decrease in food intake \\
\hline & $2=$ & no decrease in food intake \\
\hline \multirow[t]{5}{*}{ B } & \multicolumn{2}{|r|}{ Weight loss during the last 3 months } \\
\hline & $0=$ & weight loss greater than $3 \mathrm{~kg}$ \\
\hline & $1=$ & does not know \\
\hline & $2=$ & weight loss between 1 and $3 \mathrm{~kg}$ \\
\hline & $3=$ & no weight loss \\
\hline \multirow[t]{4}{*}{ C } & \multicolumn{2}{|r|}{ Mobility } \\
\hline & $0=$ & bed or chair bound \\
\hline & $1=$ & able to get out of bed/chair but does not go out \\
\hline & $2=$ & goes out \\
\hline \multirow[t]{3}{*}{ D } & \multicolumn{2}{|c|}{$\begin{array}{l}\text { Has suffered psychological stress or acute disease in the past } 3 \\
\text { months? }\end{array}$} \\
\hline & $0=$ & Yes \\
\hline & $1=$ & No \\
\hline \multirow[t]{4}{*}{$\mathbf{E}$} & \multicolumn{2}{|r|}{ Neuropsychological problems } \\
\hline & $0=$ & severe dementia or depression \\
\hline & $1=$ & mild dementia \\
\hline & $2=$ & no psychological problems \\
\hline \multirow[t]{9}{*}{$\mathbf{F}$} & \multicolumn{2}{|c|}{ Body Mass Index (BMI) (weight in kg) / (height in m2) } \\
\hline & $0=$ & BMI less than 19 \\
\hline & $1=$ & BMI 19 to less than 21 \\
\hline & $2=$ & BMI 21 to less than 23 \\
\hline & $3=$ & BMI 23 or greater \\
\hline & \multicolumn{2}{|r|}{ Screening score (max. 14 points) } \\
\hline & $12-14$ points & Normal nutritional status \\
\hline & 8-11 points & At risk of malnutrition \\
\hline & $0-7$ points & Malnourished \\
\hline
\end{tabular}




\section{Ethical approval}

This study was approved by the Institutional Ethics Review Board for Clinical Studies, Josai University (approval number: 2017-28A).

\section{Results and Discussion}

Among the 1253 (728 males, 525 females) study participants, most patients $(n=929,74.1 \%)$ were $>65$ years old (Table 4$)$. The percentage of patients increased with increasing age, as follows: $20-29$ years $(\mathrm{n}=18$, $1.4 \%)$; $30-39$ years $(n=34,2.7 \%) ; 40-49$ years $(n=89,7.1 \%) ; 50-59$ years $(\mathrm{n}=111,8.9 \%) ; 60-69$ years $(\mathrm{n}=203,16.2 \%) ; 70-79$ years $(\mathrm{n}=399$, $31.8 \%) ; 80-89$ years $(n=314,25.1 \%)$, and; $90-99$ years $(n=6.8 \%)$. The average patient weight was $53.7 \pm 14.4 \mathrm{~kg}$ and the mean BMI was 21.6 $\pm 4.6 \mathrm{~kg} / \mathrm{m}^{2}$.

The results of the nutritional risk assessment using the mSGA showed that the nutritional status of the patients was evaluated as follows: no risk $(\mathrm{n}=623 ; 49.7 \%)$; low risk $(\mathrm{n}=441 ; 35.2 \%)$; medium risk $(n=151 ; 12.1 \%)$, and; high risk $(n=38,3.0 \%)$ (Table 5$)$. Therefore, regarding the low, middle, and high risks identified in 630 patients, we focused on determining which evaluation items were responsible for these findings, and we then assessed how each of these evaluation items affected the risk assessment. The frequency for each evaluation item was identified as follows: decreased dietary intake $(n=192$ patients); weight loss ( $n=169$ patients); diarrhea and vomiting $(n=96$ patients); fever and dehydration ( $n=225$ patients); dysphagia ( $n=126$ patients); tube feeding and enteral nutrition ( $\mathrm{n}=34$ patients); decubitus ulceration ( $\mathrm{n}=48$ patients); BMI ( $\mathrm{n}=267$ patients), and; serum albumin value ( $\mathrm{n}=142$ patients) (Table 6 ). The 4 most commonly identified items, namely, decreased dietary intake, weight loss, fever and dehydration, and BMI $<18.5$ (emaciation) were found to be the key risk factors in evaluating nutritional status.

Table 4: Patient characteristics.

\begin{tabular}{|l|c|c|c|}
\hline \multirow{2}{*}{ Age } & \multicolumn{2}{|c|}{ Number of patient } & \multirow{2}{*}{ BMI (kg/m $\mathbf{~})$} \\
\cline { 2 - 3 } & $\mathbf{n}$ & $\mathbf{( \% )}$ & \\
\hline $20 \mathrm{~s}$ & 18 & $(1.4)$ & $23.4 \pm 9.0$ \\
\hline $30 \mathrm{~s}$ & 34 & $(2.7)$ & $22.7 \pm 4.3$ \\
\hline $40 \mathrm{~s}$ & 89 & $(7.1)$ & $24.8 \pm 5.4$ \\
\hline $50 \mathrm{~s}$ & 111 & $(8.9)$ & $24.5 \pm 4.8$ \\
\hline $60 \mathrm{~s}$ & 203 & $(16.2)$ & $22.2 \pm 4.3$ \\
\hline $70 \mathrm{~s}$ & 396 & $(31.6)$ & $22.6 \pm 4.5$ \\
\hline $80 \mathrm{~s}$ & 314 & $(25.1)$ & $21.2 \pm 4.0$ \\
\hline $90 \mathrm{~s}$ & 85 & $(6.8)$ & $19.4 \pm 3.5$ \\
\hline Total & 1253 & $(100)$ & $21.6 \pm 4.6$ \\
\hline
\end{tabular}

Table 5: The evaluation results concerning nutritional risk using each evaluation tool.

\begin{tabular}{|c|c|c|c|}
\hline \multirow{2}{*}{ Tool } & \multicolumn{2}{|c|}{ Routine clinical care } & Treat \\
\hline \multirow{3}{*}{ mSGA } & No risk & Low risk & Medium and High risk \\
\cline { 2 - 4 } & 623 & 441 & 189 \\
\cline { 2 - 4 } & $(49.7 \%)$ & $(35.2 \%)$ & $(15.1 \%)$ \\
\hline \multirow{3}{*}{ MUST } & Score 0 & Score 1 & Score 2 \\
\cline { 2 - 4 } & 684 & 176 & 393 \\
\cline { 2 - 4 } & $(54.6 \%)$ & $(14.0 \%)$ & $(31.4 \%)$ \\
\hline \multirow{3}{*}{ MNA - -SF } & Normal & At risk & Malnourished \\
\cline { 2 - 4 } & 491 & 459 & 303 \\
\cline { 2 - 4 } & $(39.2 \%)$ & $(36.6 \%)$ & $(24.2 \%)$ \\
\hline
\end{tabular}

A breakdown of each of these 4 risk factors showed the following: food loss was low risk $(n=86)$; medium risk $(n=83)$, or; high risk $(n=23)$. Weight loss was low risk $(n=78)$; medium risk $(n=68)$, or; high risk $(n=23)$. Fever and dehydration was low risk $(n=116)$; medium risk $(\mathrm{n}=82)$, or; high risk $(\mathrm{n}=27)$. BMI $<18.5$ (emaciation) was low risk $(n=54)$; medium risk, $(n=81$, or; high risk $(n=32)$. These 4 items were reconfirmed as important factors in relation to the mSGA endpoint. It has previously been reported that a BMI $<18.5$ (emaciation) is associated with an increased risk of developing pressure ulcers [18], decreased immunity [19], and a decreased self-sustaining capacity to engage in activities of daily life due to muscle weakness [20] in elderly people. Given that patients who were elderly accounted for $>70 \%$ of the total number of patients in this study, a BMI $<18.5$ (emaciation) could be considered an important assessment item (Tables 4 and 5).

We then examined the assessment utilization rate of each evaluation item for each risk level (Table 6). In 38 high-risk patients, the following results were obtained: BMI, $\mathrm{n}=32(84 \%)$; decubitus ulceration, $\mathrm{n}=28$ (74\%); fever and dehydration, $\mathrm{n}=27$ (71\%); dysphagia, $\mathrm{n}=24(63 \%)$; decreased dietary intake, $n=23$ (61\%); body weight loss, $n=23(61 \%)$, and; serum albumin level, $\mathrm{n}=23$ (61\%). Among 151 medium-risk patients, the following results were obtained: dietary loss, $n=83$ (55\%); BMI <18.5(emaciation), $\mathrm{n}=81$ (54\%); fever and dehydration, $\mathrm{n}=82$ (54\%), and; weight loss, $\mathrm{n}=68$ (45\%). Among 151 medium-risk patients, the following results were obtained: dietary loss, $n=83(55 \%)$; BMI <18.5 (emaciation), $\mathrm{n}=81$ (54\%); fever and dehydration, $\mathrm{n}=82$ (54\%), and; weight loss, $\mathrm{n}=68$ (45\%). Interestingly, it was confirmed that, among the initial nutritional status evaluation items, decubitus ulceration and serum albumin levels were frequently identified in addition to the 4 items previously identified, such as a BMI $<18.5$ (emaciation). We found that there was a high number of patients with decubitus ulcers (pressure ulcers) evaluated as high risk compared to medium risk.

Evaluation using the SGA confirmed the importance of factors such as BMI, decubitus ulceration, and weight loss. Therefore, we examined the relationship between each assessment factor and age groups (Table 7).

Among those aged from 20 to 39 years (52 patients), we found that fever and dehydration ( $\mathrm{n}=13$ patients, $25.0 \%)$, and diarrhea and vomiting $(\mathrm{n}=10$ patients, $19.2 \%)$ evaluation items were frequently involved. The younger age group appeared to have more acute than chronic cases.

Among patients aged from 40 to 64 years ( $n=272$ patients), the importance of the following evaluation items concerning decreased dietary intake $(\mathrm{n}=34,12.5 \%), \mathrm{BMI}(\mathrm{n}=39,14.3 \%)$, and fever and dehydration $(\mathrm{n}=35,12.9 \%)$ was shown. Furthermore, in elderly people $>65$ years $(n=929)$, the importance of the following evaluation items concerning BMI $(n=220,23.7 \%)$, fever and dehydration $(n=177$, $19.1 \%)$, decreased dietary intake $(n=157,16.9 \%)$, weight loss $(n=138$, $14.9 \%)$, serum albumin level $(\mathrm{n}=118,12.7 \%)$, and decubitus ulceration $(\mathrm{n}=46,5.0 \%)$ was shown. The items concerning meal reduction showed closely related values, with only a difference of approximately $4 \%$ found between those aged from 40 to 64 years and those aged over 65 years. However, a difference of approximately $10 \%$ was found in BMI values between elderly people (those aged $>65$ years) and those aged from 40 to 64 years. Additionally, it was found that a large percentage of the elderly patients had decubitus ulcers.

One reason for the decrease in BMI was because of a decrease in albumin levels, due to a decrease in protein intake in elderly people [21], and a decrease in muscle mass [22]. The daily intake of meat

Citation: Araki J, Inoue Y, Honda L, Morita S, Tsuchiya M, et al. (2020) Nutritional Risk Assessment at a Municipal Hospital Using Nutritional 
Table 6: The status of the mSGA evaluation items classified according to each nutritional risk factor.

\begin{tabular}{|c|c|c|c|c|c|c|c|c|c|c|}
\hline \multirow[t]{2}{*}{ mSGA Content } & \multicolumn{2}{|c|}{$\begin{array}{l}\text { No risk } \\
(n=623)\end{array}$} & \multicolumn{2}{|c|}{$\begin{array}{l}\text { Low risk } \\
(n=441)\end{array}$} & \multicolumn{2}{|c|}{$\begin{array}{l}\text { Medium risk } \\
\quad(n=151)\end{array}$} & \multicolumn{2}{|c|}{$\begin{array}{l}\text { High risk } \\
(n=38)\end{array}$} & \multicolumn{2}{|c|}{$\begin{array}{c}\text { Total } \\
(n=1253)\end{array}$} \\
\hline & $n$ & $(\%)$ & $\mathbf{n}$ & $(\%)$ & $\mathbf{n}$ & (\%) & 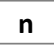 & (\%) & $\mathbf{n}$ & $(\%)$ \\
\hline Decrease in food intake & 0 & $(0.0)$ & 86 & $(19.5)$ & 83 & $(55.0)$ & 26 & (68.4) & 195 & $(15.6)$ \\
\hline Weight loss & 0 & $(0.0)$ & 78 & (17.7) & 68 & $(45.0)$ & 23 & $(60.5)$ & 169 & (13.5) \\
\hline Diarrhea/Vomiting & 0 & $(0.0)$ & 55 & $(12.5)$ & 33 & (21.9) & 8 & $(21.1)$ & 96 & $(7.7)$ \\
\hline Fever/Dehydration & 0 & $(0.0)$ & 116 & $(26.3)$ & 82 & (54.3) & 27 & (71.1) & 225 & (18.0) \\
\hline Dysphagia & 0 & $(0.0)$ & 41 & $(9.3)$ & 61 & $(40.4)$ & 24 & $(63.2)$ & 126 & $(10.1)$ \\
\hline Tube feeding & 0 & $(0.0)$ & 10 & $(2.3)$ & 13 & $(8.6)$ & 11 & $(28.9)$ & 34 & $(2.7)$ \\
\hline Pressure ulcers & 0 & $(0.0)$ & 0 & $(0.0)$ & 20 & $(13.2)$ & 28 & (73.7) & 48 & (3.8) \\
\hline $\mathrm{BMI}<18.5 \mathrm{~kg} / \mathrm{m}^{2}$ & 0 & $(0.0)$ & 154 & $(34.9)$ & 81 & $(53.6)$ & 32 & $(84.2)$ & 267 & $(21.3)$ \\
\hline Serum albumin value $<3.0$ & 0 & $(0.0)$ & 69 & $(15.6)$ & 50 & (33.1) & 23 & $(60.5)$ & 142 & (11.3) \\
\hline
\end{tabular}

Table 7: The status of the mSGA evaluation items classified according to age group.

\begin{tabular}{|c|c|c|c|c|c|c|c|c|}
\hline \multirow[t]{2}{*}{ mSGA content } & \multicolumn{2}{|c|}{$\begin{array}{c}20-39 \text { years } \\
\quad(n=52)\end{array}$} & \multicolumn{2}{|c|}{$\begin{array}{c}\text { 40-64 years } \\
(n=272)\end{array}$} & \multicolumn{2}{|c|}{$\begin{array}{l}\text { over } 65 \text { years } \\
\qquad(n=929)\end{array}$} & \multicolumn{2}{|c|}{$\begin{array}{c}\text { Total } \\
(n=1253)\end{array}$} \\
\hline & $\mathrm{n}$ & (\%) & $\mathrm{n}$ & (\%) & $n$ & (\%) & $\mathbf{n}$ & (\%) \\
\hline Decrease in food intake & 4 & (7.7) & 34 & $(12.5)$ & 157 & (16.9) & 195 & $(15.6)$ \\
\hline Weight loss & 4 & $(7.7)$ & 27 & (9.9) & 138 & (14.9) & 169 & $(13.5)$ \\
\hline Diarrhea/ Vomiting & 10 & (19.2) & 26 & $(9.6)$ & 60 & $(6.5)$ & 96 & $(7.7)$ \\
\hline Fever /Dehydration & 13 & $(25.0)$ & 35 & (12.9) & 177 & (19.1) & 225 & $(18.0)$ \\
\hline Dysphagia & 1 & $(1.9)$ & 10 & $(3.7)$ & 115 & (12.4) & 126 & $(10.1)$ \\
\hline Tube feeding & 1 & (1.9) & 4 & $(1.5)$ & 29 & (3.1) & 34 & $(2.7)$ \\
\hline Pressure ulcers & 0 & $(0.0)$ & 2 & $(0.7)$ & 46 & $(5.0)$ & 48 & $(3.8)$ \\
\hline $\mathrm{BMI}<18.5 \mathrm{~kg} / \mathrm{m}^{2}$ & 8 & $(15.4)$ & 39 & $(14.3)$ & 220 & $(23.7)$ & 267 & $(21.3)$ \\
\hline Serum albumin value $<3.0$ & 2 & $(3.8)$ & 22 & $(8.1)$ & 118 & $(12.7)$ & 142 & (11.3) \\
\hline
\end{tabular}

Table 8: The status of the MUST evaluation items classified according to each nutritional risk factor.

\begin{tabular}{|l|c|c|c|c|c|c|c|c|}
\hline \multirow{2}{*}{$\begin{array}{c}\text { MUST } \\
\text { content }\end{array}$} & \multicolumn{2}{c|}{$\begin{array}{c}\text { Score 0 } \\
(\mathbf{n}=\mathbf{6 8 4}\end{array}$} & \multicolumn{2}{c|}{$\begin{array}{c}\text { Score 1 } \\
\mathbf{( n = 1 7 6 )}\end{array}$} & \multicolumn{2}{c|}{$\begin{array}{c}\text { Score 2 or } \\
\text { more }(\mathbf{n = 3 9 3 )}\end{array}$} & \multicolumn{2}{c|}{$\begin{array}{c}\text { Total } \\
(\mathbf{n}=1253)\end{array}$} \\
\cline { 2 - 9 } & $\mathbf{n}$ & $\mathbf{( \% )}$ & $\mathbf{n}$ & $\mathbf{( \% )}$ & $\mathbf{n}$ & $\mathbf{( \% )}$ & $\mathbf{n}$ & $(\%)$ \\
\hline BMI & 0 & 0 & 118 & $(67.0)$ & 306 & $(77.9)$ & 424 & $(33.8)$ \\
\hline $\begin{array}{l}\text { Weight } \\
\text { loss }\end{array}$ & 0 & 0 & 58 & $(33.0)$ & 111 & $(28.2)$ & 169 & $(13.5)$ \\
\hline $\begin{array}{l}\text { Acute } \\
\text { disease } \\
\text { effect }\end{array}$ & 0 & 0 & 0 & 0 & 195 & $(49.6)$ & 195 & $(15.6)$ \\
\hline
\end{tabular}

among Japanese people has been reported to decrease as they become older [23]. In general, the serum albumin level, which is a nutritional index of the body, has a positive relationship with body muscle mass. Older people with a better nutritional status and more muscle mass are less likely to lose their ability to walk with age. Moreover, decubitus ulceration has been reported to be associated with serum albumin levels and is implicated in the nutritional status of elderly people [24]; therefore, it is evident that diet content greatly affects the nutritional status. It is necessary to improve the nutritional status of elderly patients through reviewing their diet content and incorporating large amount of proteins.

However, the evaluation rates of serum albumin levels were found to be similar among those aged from 40 to 64 years and among those aged $\geq 65$ years. In general, concerning elderly people, basal metabolism decreases due to aging, with less fluid produced during metabolism, which increases the likelihood of dehydration. Therefore, it is possible that the serum albumin levels among the elderly patients could have increased due to this factor.

For the above reasons, this study evaluated not only the serum albumin level, but also considered a bodily assessment involving decubitus ulceration in relation to nutritional status (Tables 6 and 7).

Dysphagia was the next most frequently used endpoint after BMI $<18.5$ (emaciation). Dysphagia is a risk factor for malnutrition as it reduces the ability to eat effectively. Therefore, an NST intervention for patients with dysphagia should focus on increasing energy and protein intake and encouraging weight increases. Of 126 patients in whom the presence of dysphagia was used for nutritional assessment, 115 involved elderly people. In elderly people, it can be expected that a decrease in the ability to swallow, a decrease in food intake, and the associated malnutrition will cause a decrease in muscle strength $[25,26]$. Dysphagia was an important item to evaluate, as $>70 \%$ of the patients in this study were elderly people.

\section{Nutritional risk assessment evaluation using the MUST}

We considered that it was important to be able to easily identify patients through nutritional screening, for effective NST intervention on initial hospital admission. We conducted a nutritional risk assessment using the MUST, which has fewer items than the mSGA (Table 2). The MUST is used to assess nutritional status according to BMI, weight loss, and decreased dietary intake. Since BMI, weight loss, and decreased dietary intake items largely corresponded to those in the mSGA, in our study we used not only the number of items but also the evaluation content of the mSGA as a reasonable comparison.

In the MUST, a score of 0 indicates that standard patient management must be performed; a score of 1 indicates that follow-ups 
Table 9: The status of the $M N A^{\circledR}$-SF evaluation items classified according to each nutritional risk factor.

\begin{tabular}{|c|c|c|c|c|c|c|c|c|}
\hline \multirow[t]{2}{*}{ MNA $^{\circledR}$-SF content } & \multicolumn{2}{|c|}{$\begin{array}{l}\text { Normal nutritional } \\
(n=491)\end{array}$} & \multicolumn{2}{|c|}{$\begin{array}{l}\text { At risk of malnutrition } \\
\qquad(n=459)\end{array}$} & \multicolumn{2}{|c|}{$\begin{array}{l}\text { Malnourished } \\
(n=303)\end{array}$} & \multicolumn{2}{|c|}{$\begin{array}{c}\text { Total } \\
(n=1253)\end{array}$} \\
\hline & $\mathbf{n}$ & $(\%)$ & $\mathbf{n}$ & $(\%)$ & $\mathbf{n}$ & $(\%)$ & $\mathbf{n}$ & $(\%)$ \\
\hline Decrease in food intake & 2 & 0.4 & 60 & 13.1 & 215 & 71 & 277 & 22.1 \\
\hline Weight loss & 0 & 0 & 50 & 10.9 & 123 & 40.6 & 173 & 13.8 \\
\hline Mobility & 114 & 23.2 & 295 & 64.3 & 271 & 89.4 & 680 & 54.3 \\
\hline $\begin{array}{l}\text { psychological stress/acute } \\
\text { disease }\end{array}$ & 15 & 3.1 & 96 & 20.9 & 183 & 60.4 & 294 & 23.5 \\
\hline Neuropsychological problems & 29 & 5.9 & 150 & 32.6 & 200 & 66 & 379 & 30.2 \\
\hline BMI & 171 & 34.8 & 350 & 76.3 & 264 & 87.1 & 785 & 62.6 \\
\hline
\end{tabular}

must be done, and a score of 2 indicates that nutritional management is necessary through NST intervention. The evaluated nutritional risk levels according to the MUST were as follows: score 0,684 patients (54.6\%); score 1,176 patients (14.0\%), and; score 2, 393 patients (31.4\%) (Table 5). The number of patients requiring intervention according to the MUST showed an increase of 204 patients compared to the 189 patients identified according to the mSGA evaluation (medium risk and high risk).

The reason for the increase in patients requiring intervention in the MUST appeared to be due to: 1) the difference in the total score for NST, and 2) the difference in the score allocation of each item. For a difference in the total score to require an NST intervention for a patient, nutritional evaluation using the mSGA leads to an intervention if decubitus ulceration is included or $\geq 3$ other items are applicable. However, in contrast with the mSGA, the MUST requires that, in relation to the 3 nutritional evaluation items (BMI, weight loss, and decreased dietary intake), an intervention must occur even if only one of these evaluation items is applicable.

We next focused on the difference in the score distributions of each item. Step 1 for the evaluation of BMI was as follows: $20 \mathrm{~kg} / \mathrm{m}^{2}$ or more, 0 points; 18.5 to $20 \mathrm{~kg} / \mathrm{m}^{2}, 1$ point, and; less than $18.5 \mathrm{~kg} /$ $\mathrm{m}^{2}, 2$ points. However, the mSGA evaluates BMI as: BMI $<18.5 \mathrm{~kg} /$ $\mathrm{m}^{2}$ (emaciation), 1 point. A total of 267 patients that corresponded to a $\mathrm{BMI}<18.5 \mathrm{~kg} / \mathrm{m}^{2}$ (emaciation) using the $\mathrm{mSGA}$ nutritional risk assessment remained subject to NST intervention when assessed using the MUST (Table 8).

In addition, a further point is added in the MUST when the BMI ranges from 18.5 to $20 \mathrm{~kg} / \mathrm{m}^{2}$. The average BMI of the patients in this study was $21.6 \pm 4.6 \mathrm{~kg} / \mathrm{m}^{2}$, which was very close to the evaluation value of the MUST. The average BMI for adults in Japan is $23.8 \pm 3.4$ $\mathrm{kg} / \mathrm{m}^{2}$ for men and $22.6 \pm 3.7 \mathrm{~kg} / \mathrm{m}^{2}$ for women [27]. It can be assumed that BMI evaluation in the MUST is likely to be a key factor in the assessment of the nutritional status of Japanese people. The MUST guidelines issued by BAPEN cite serious cases such as dysphagia due to cerebral infarction and head injury as an example of the MUST Step 3 (food intake situation) [10]. In this study, where dysphagia was determined as corresponding to the item "decreased food intake" in the mSGA, many more patients were confirmed as requiring intervention for malnutrition as this factor conformed with the MUST Step 3. As Step 3 may be applicable to dysphagia, including it as required in the MUST evaluation is likely to increase the number of cases requiring intervention. Thus, it could be seen that the MUST presents difficulties as an evaluation tool when used to extrapolate data from the mSGA due to the unmatched character of the evaluation items (Table 8).

\section{Nutritional risk assessment using the $\mathrm{MNA}^{\oplus}-\mathrm{SF}$}

Among the 1,253 participants in this study, 929 patients (74.1\%) were elderly people. Therefore, the nutritional risk was evaluated using the $\mathrm{MNA}^{\oplus}-\mathrm{SF}$, which is a simplified version of the MNA created specifically for assessing elderly people. The results of nutritional risk assessment using the $\mathrm{MNA}^{\oplus}$-SF were categorized as follows: good nutrition status, $\mathrm{n}=491(39.2 \%)$; at risk, $\mathrm{n}=459(36.6 \%)$, and; malnutrition targeted for NST intervention, $\mathrm{n}=303(24.2 \%)$ (Table 5).

Compared to the risk assessment results using the mSGA, the MNA $^{\circ}$-SF results showed fluctuations in nutritional risk (Scheme 1). Scheme 1 shows the nutritional risk variation of $\mathrm{MNA}^{\circ}-\mathrm{SF}$ and MUST compared to mSGA. When examining the results in greater detail, it was found that 24 of 189 patients determined in the mSGA as requiring intervention were assessed in the $\mathrm{MNA}^{\circ}-\mathrm{SF}$ as patients not requiring intervention ( $n=1$ patient, good nutrition; $n=23$ patients, at risk). In addition, 2 patients assessed as without risk and not requiring an intervention and 136 patients assessed as low risk in the mSGA were determined to require intervention for malnutrition as a result of nutritional risk determination using the $\mathrm{MNA}^{\oplus}$-SF. To explain these risk assessment fluctuations, them SGA included decubitus ulceration as an evaluation item whereas the $\mathrm{MNA}^{\circ}$-SF focused more on evaluating the degree of independence and cognitive function in relation to daily life.

\section{The results of using the $\mathrm{MNA}^{\circledast}$-SF evaluation items}

The total number of patient evaluations for each assessment item was: decreased food intake (277 patients); weight loss (173 patients); walking unaided (680 patients); acute disease (294 patients); presence or absence of neurological and mental issues (379 patients), and BMI (785 patients) (Table 8 ). As a result, the 3 items 'walking unaided', 'presence or absence of neurological and mental problems', and 'BMI' were shown to be the major risk factors. The characteristic feature of the $\mathrm{MNA}^{\oplus}$-SF created for elderly people was shown to be its capacity to evaluate factors such as walking unaided, the presence or absence of neurological and mental problems, and BMI.

Since $\geq 70 \%$ of the patients in this study were elderly, the major items of the $\mathrm{MNA}^{\circ}$-SF were often evaluated. Moreover, in the MNA ${ }^{\circ}$ SF, BMI was divided as follows: 0 points, $<19 \mathrm{~kg} / \mathrm{m}^{2} ; 1$ point, $\geq 19 \mathrm{~kg} /$ $\mathrm{m}^{2}$ but $<21 \mathrm{~kg} / \mathrm{m}^{2} ; 2$ points, $\geq 21 \mathrm{~kg} / \mathrm{m}^{2}$ but $<23 \mathrm{~kg} / \mathrm{m}^{2}$, and; 3 points, $\geq 23 \mathrm{~kg} / \mathrm{m}^{2}$, to evaluate 4 possible levels. As mentioned above, the average BMI for Japanese men is $23.7 \pm 3.2 \mathrm{~kg} / \mathrm{m}^{2}$ and $22.4 \pm 3.4 \mathrm{~kg} / \mathrm{m}^{2}$ for women [24], while the average BMI for the patients in this study was $21.6 \pm 4.6 \mathrm{~kg} / \mathrm{m}^{2}$. Therefore, $>50 \%$ of the patients had BMI values closely aligned with the evaluation value of the $\mathrm{MNA}^{\circ}-\mathrm{SF}$. 
Furthermore, we examined the evaluation utilization rate of each item in relation to each risk factor (Table 9). A breakdown of the risk factors that necessitated malnutrition intervention showed the following for 303 patients: food intake reduction, 215 (71\%); weight loss, 123 (41\%); walking unaided, 271 (89\%); acute disease, $183(60 \%)$; neurological and mental issues, 200 (66\%), and; BMI, 264 (87\%). Among the 459 patients identified as at risk, the following items were determined: dietary intake reduction, 60 (13\%); weight loss, 50 (11\%); walking unaided, 295 (64\%); acute disease, 96 (21\%); neurological and mental issues, 150 (33\%), and; BMI, 350 (76\%). As a result, it was revealed that walking unaided and BMI were major evaluation risk factors of the nutritional risk level. Moreover, differences in the number of evaluations were found depending on the nutritional status in relation to decreased food intake, weight loss, acute disease, and neurological and mental issues. Decreased food intake, weight loss, and acute disease were identified as major evaluation risk factors likely to require intervention for patients in the $\mathrm{mSGA}$ and were important factors in nutritional evaluation in the $\mathrm{MNA}^{\bullet}$-SF.

The $\mathrm{MNA}^{\oplus}$-SF is designed to assess the nutritional status of frail elderly people. Sarcopenia and frailty are also major issues among elderly people. In particular, a frailty cycle brought on through a decrease in food intake, malnutrition, decreased muscle mass, decreased basal metabolism, and decreased energy expenditure is a serious problem. Elderly patients are more likely to have sarcopenia and frailty because they are, on average, less able to walk unaided and engage in necessary physical activity (Table 9).

BMI is an important predictor of mortality regardless of age or sex [28]. Patients with a low BMI also have an increased incidence of decubitus ulceration to the sacrum, sciatic, hip, and shoulder regions [18]. There have been various reports on the relationship between BMI and decubitus ulceration incidence [29,30]. In addition, while the average BMI of Japanese people is close to the $\mathrm{MNA}^{\circ}$-SF evaluation value, working within that evaluation value appears likely to be appropriate in helping to prevent the occurrence of decubitus ulceration.

The MUST is designed to help distinguish between underweight and malnourished adults and obese people. In addition, there are only 3 evaluation items, namely: BMI, weight loss and reduced dietary intake, which makes it a very simple evaluation tool to use. When assessing nutritional risk using the MUST, the number of interventions required was found to double compared to the mSGA. Despite this increase in the number of interventions, 28 of 189 interventions in the mSGA were determined as not needing NST interventions in the MUST (Scheme 1). The patients in this study were mostly elderly people, and patients who need to use physical assessment in relation to issues such as decubitus ulceration and dysphagia for nutritional assessment tend not to be included for intervention.

Nutritional risk assessment using mSGA, MUST, and MNA ${ }^{\circ}$-SF indicates that $\mathrm{MNA}^{\circ}-\mathrm{SF}$ appears to be a reliable assessment tool and may be applicable to patients in this study. Its advantages for use were as follows: 1) fewer evaluation items compared to the mSGA; 2) an option of "don't know" in weight loss evaluation; 3) applicable to elderly patients who comprised a majority of the study population, and; 4) data involving patient intervention for young people extractable. In particular, the "don't know" option in weight loss evaluation leads to a simple evaluation method in hospitals and at home, because it is possible to perform nutritional assessment even in environments that can be difficult to operate within due to a patient's reduced level of awareness and that of their family and care givers.
The $\mathrm{MNA}^{\oplus}$-SF does not use clinical laboratory data, such as serum albumin levels. Moreover, the calf circumference can be used as an alternative measurement if the BMI cannot be measured $[15,31]$. Therefore, the $\mathrm{MNA}^{\oplus}-\mathrm{SF}$ can be easily undertaken at a patient's home.

In community healthcare in Japan, the number of patients receiving home care is increasing yearly, and exceeded 180,000 in 2017 [32]. In Japan, the number of pharmacies that undertake home visit interventions is also increasing rapidly, and this provides pharmacists with opportunities to assist in nutritional support. Evaluating a patient's status in terms of diet history, weight fluctuation, cognitive function and stress condition can be undertaken by family members at home, as well as by doctors, nurses, pharmacists, and caregivers who visit regularly. If the same nutrition assessment tool is used not only at the municipal hospital but also at home care in the community, it will facilitate the provision of necessary information for screening on initial admission and help to ensure readily determinable and appropriate nutritional assessment. We consider that pharmacists need to be involved in nutritional assessment as part of the collaborative efforts of healthcare teams to help link hospital and home services more effectively.

\section{Acknowledgement}

The authors wish to sincerely thank all the subjects for their participation in this study.

\section{Conflict of Interest}

The authors declare that there are no conflicts of interests regarding the publication of this paper.

\section{References}

1. Haydock DA, Hill GL (1986) Impaired wound healing in surgical patients with varying degrees of malnutrition. J Parenter Enter Nutr 10: 550-554.

2. Agarwal E, Ferguson M, Banks M, Batterham M, Bauer J, et al. (2013) Malnutrition and poor food intake are associated with prolonged hospital stay, frequent readmissions, and greater in-hospital mortality: results from the Nutrition Care Day Survey 2010. Clin Nutr 32: 737-745.

3. Ruiz AJ, Buitrago G, Rodríguez N, Gómez G, Sulo S, et al. (2019) Clinical and economic outcomes associated with malnutrition in hospitalized patients. Clin Nutr 38: 1310-1316.

4. Cong MH, Li SL, Cheng GW, Liu JY, Song CX, et al. (2015) An Interdisciplinary Nutrition Support Team Improves Clinical and Hospitalized Outcomes of Esophageal Cancer Patients with Concurrent Chemoradiotherapy. Chin Med J (Engl) 128: 3003-3007.

5. Park YE, Park SJ, Park Y, Cheon JH, Kim TI, et al. (2017) Impact and outcomes of nutritional support team intervention in patients with gastrointestinal disease in the intensive care unit. Medicine (Baltimore) 96: e8776.

6. Detsky AS, McLaughlin JR, Baker JP, Johnston N, Whittaker S, et al. (1987) What is subjective global assessment of nutritional status? J Parenter Enteral Nutr 11: 8-13.

7. Sungurtekin H, Sungurtekin U, Balci C, Zencir M, Erdem E (2004) The influence of nutritional status on complications after major intraabdominal surgery. J Am Coll Nutr 23: 227-232.

8. Pham NV, Cox-reijven PL, Greve JW, Soeters PB (2006) Application of subjective global assessment as a screening tool for malnutrition in surgical patients in Vietnam. Clin Nutr 25: 102-108. 
9. Kang MC, Kim JH, Ryu SW, Moon JY, Park JH, et al. (2018) Prevalence of Malnutrition in Hospitalized Patients: a Multicenter Crosssectional Study. J Korean Med Sci 33: e10.

10. Malnutrition Action Group (2010) THE 'MUST' EXPLANATORY BOOKLET. BAPEN.

11. Stratton RJ, King CL, Stroud MA, Jackson AA, Elia M (2006) 'Malnutrition Universal Screening Tool' predicts mortality and length of hospital stay in acutely ill elderly. Br J Nutr 95: 325-330.

12. Kruizenga $\mathrm{H}$, van Keeken S, Weijs $\mathrm{P}$, Bastiaanse L, Beijer S, et al. (2016) Undernutrition screening survey in 564,063 patients: patients with a positive undernutrition screening score stay in hospital $1.4 \mathrm{~d}$ longer. Am J clin Nutr 103: 1026-1032.

13. Guigoz Y, Vellas B, Garry PJ (1996) Assessing the nutritional status of the elderly: The Mini Nutritional Assessment as part of the geriatric evaluation. Nutr Rev 54: S59-S65.

14. Rubenstein LZ, Harker JO, Salvà A, Guigoz Y, Vellas B (2001) Screening for undernutrition in Geriatric Practice: developing the short-form mini-nutritional assessment (MNA-SF). J Gerontol A Biol sci Med Sci 56: M366-M372.

15. Dent E, Chapman I, Piantadosi C, Visvanathan R (2017) Screening for malnutrition in hospitalised older people: Comparison of the Mini Nutritional Assessment with its short-form versions. Australas J Ageing 36: E8-E13.

16. Sato K (2016) Mini Nutritional Assessment Short-Form (MNA-SF) Predicts Clinical Outcomes: Cohort Study of Small-Sized Hospital in Japan. J Gen Fam Med 17: 90-98.

17. Kuzuya M, Kanda S, Koike T, Suzuki Y, Satake S, et al. (2005) Evaluation of Mini-Nutritional Assessment for Japanese frail elderly. Nutrition 21: 498-503.

18. Kottner J, Gefen A, Lahmann N (2011) Weight and pressure ulcer occurrence: a secondary data analysis. Int J Nurs Stud 48: 13391348.

19. Ilavská $\mathrm{S}$, Horváthová $\mathrm{M}$, Szabová $\mathrm{M}$, Nemessányi $\mathrm{T}$, Jahnová $\mathrm{E}$, et al. (2012) Association between the human immune response and body mass index. Hum Immunol 73: 480-485.

20. Bürge E, Berchtold A, von Gunten A (2011) Gender-related ADL performance of old people recently admitted to a Swiss nursing home. A cross-sectional study. Swiss Med Wkly 141: w13183.
21. Madhuvanthi M, Lathadevi GV (2016) Serum Proteins Alteration in Association with Body Mass Index in Human Volunteers. J Clin Diagn Res 10: CC05-CCO7.

22. Graf CE, Pichard C, Herrmann FR, Sieber CC, Zekry D, et al. (2017) Prevalence of low muscle mass according to body mass index in older adults. Nutrition 34: 124-129.

23. National Institute of Health and Nutrition (2018) National Health and Nutrition Survey.

24. Neloska L, Damevska K, Nikolchev A, Pavleska L, Petreska-Zovic B, et al. (2016) The Association between Malnutrition and Pressure Ulcers in Elderly in Long-Term Care Facility. Open Access Maced J Med Sci 4: 423-427.

25. Tagliaferri S, Lauretani F, Pelá G, Meschi T, Maggio M (2019) The risk of dysphagia is associated with malnutrition and poor functional outcomes in a large population of outpatient older individuals. Clin Nutr 38: 2684-2689.

26. Idah Chatindiara, Jacqueline Allen, Amy Popman, Darshan Patel, Marilize Richter, et al. (2018) Dysphagia risk, low muscle strength and poor cognition predict malnutrition risk in older adults at hospital admission. BMC Geriatr 18: 78.

27. National Institute of Health and Nutrition (2018) National Health and Nutrition Survey, Physical Status Questionnaire.

28. Landi F, Onder G, Gambassi G, Pedone C, Carbonin P, et al. (2000) Body mass index and mortality among hospitalized patients. Arch Intern Med 160: 2641-2644.

29. Hyun S, Li X, Vermillion B, Newton C, Fall M, et al. (2014) Body mass index and pressure ulcers: improved predictability of pressure ulcers in intensive care patients. Am J Crit Care 23: 494-500.

30. Cai S, Rahman M, Intrator O (2013) Obesity and pressure ulcers among nursing home residents. Med Care 51: 478-486.

31. Montejano Lozoya R, Martínez-Alzamora N, Clemente Marín G, Guirao-Goris SJA, Ferrer-Diego RM (2017) Predictive ability of the Mini Nutritional Assessment Short Form (MNA-SF) in a free-living elderly population: a cross-sectional study. PeerJ 5: e3345.

32. Ministry of Health, Labour and Welfare (2018) Patient Survey. 